\title{
Creating and Modelling Personal Socio-Economic Networks in On-Line Banking
}

\author{
Beatriz San Miguel , Jose M. del Alamo, Juan C. Yelmo
}

\begin{abstract}
The banking industry is observing how new competitors threaten its millennial business model by targeting unbanked people, offering new financial services to their customer base, and even enabling new channels for existing services and customers. The knowledge on users, their behaviour, and expectations become a key asset in this new context. Well aware of this situation, the Center for Open Middleware, a joint technology center created by Santander Bank and Universidad Politécnica de Madrid, has launched a set of initiatives to allow the experimental analysis and management of socio-economic information. PosdataP2P service is one of them, which seeks to model the economic ties between the holders of university smart cards, leveraging on the social networks the holders are subscribed to. In this paper we describe the design principles guiding the development of the system, its architecture and some implementation details.
\end{abstract}

Keywords: social networks, on-line banking, user modelling, ontology

\section{$1 \quad$ Introduction}

Detailed information about customers and users has become a critical asset for most organizations. Context and personal data give them "the ability to understand and even predict where humans focus their attention and activity at the individual, group and global level" [1]. Companies, governments and other organizations can leverage on these data to improve their products, boost their users' experience, personalize their services, develop and deliver new products tailored to their customers' needs and expectations, fidelize existing customers and attract new ones, etc. On-line banking is not an exception and, for some time now, banks have been collecting and processing information about their customers to improve their traditional services.

However, new entrants in the domain threaten traditional retail and commercial online banking business models, by leveraging on their proficiency in technology and knowledge generation. Inevitably, "...banks that are not prepared for such new competitors face certain death" [2]. Furthermore, some studies call for a business model switch towards user-centricity that consistently puts the customer at the heart of all activities [3]. This is especially important in some regions were unbanked people 
(those adults who do not use traditional financial services) reach an astonishing $65 \%$ of the population [4].

Well aware of this situation, the Center for Open Middleware (COM), a joint technology center created in 2011 by Santander Bank and Universidad Politécnica de Madrid, has launched some initiatives aimed at meeting the aforementioned challenges. COM is the incubator of an open software ecosystem developing middleware solutions and experimenting with new software approaches. Specifically, the POSDATA (Personal and Social Data Analysis) project provides a conceptual framework and technology support to allow the experimental analysis and management of personal and social data of university students with the goal of personalizing, and thus improving, the services provided by universities and Santander Bank.

In this paper we introduce an innovative service developed within the POSDATA framework, namely PosdataP2P (Person-to-Person), which enables issuing payments to and demanding money from the student's social relationships. With the goal of gaining knowledge on this users' base, we characterize the PosdataP2P users, by proposing a model that includes raw personal data generated by our service as well as other data available at the user's social networks.

The structure of this paper is organized as follows. The next section briefly introduces the POSDATA project and the PosdataP2P service. Then, section 3 provides a background regarding personal data and its associated terminology, and section 4 discusses the current challenges in the user modelling process. After that, we present the system architecture of our solution, describing its components and relevant processes in section 5, and we give some implementation details in section 6. Finally, section 7 analyses the related work, and section 8 concludes the paper.

\section{The POSDATA Project}

The POSDATA project provides a conceptual framework and technology support to allow the experimental analysis and management of personal and social data of the Santander University Smart Card (USC) holders [5].

The USC is a smart card issued by different universities in collaboration with Santander Bank. It can be used as a personal identification enabling services such as the electronic signature, access control to restricted areas, library loans, request of academic files, discounts in different associated businesses, and many other services. The USC holders can top-up their cards, so that they are able to use services such as vending machines or photocopiers. Optionally, the USC can be enabled to gain access to Santander Bank financial services, working as a credit/debit card linked to the holder's saving account. The USC is currently used by almost 6,3 million people in over 262 universities worldwide.

Leveraged by the POSDATA goals of gaining knowledge on these students in a banking context, an experimental service (currently a prototype), called PosdataP2P, has been developed. PosdataP2P service allows USC holders to make payments to or demand money from their friends using alternative social channels such as texting systems e.g. Telegram, or online social networks e.g. Facebook or Twitter. 
To use PosdataP2P service, USC holders have to activate the service first, providing their USC information. Then, they choose the social channels that they want to use to carry out financial transactions. The PosdataP2P website supports USC holders in these steps, and additionally provides them with the terms of service and privacy policy, details on their current balance and recent activities, information on the social channels they have enabled, and so on.

Once PosdataP2P service is activated and configured, its users can start making financial transactions by simply posting messages to their friends within their enabled social channels. The only restrictions imposed by PosdataP2P are that 1) the user issuing a payment must have a balance equal or greater than the amount being transferred; and, 2) the texts used to trigger the transactions must follow a predefined pattern to be understood by the service. This pattern includes four basic elements:

- the identification of the terminator of the transaction i.e. the friend who receives the money or from whom the money is demanded;

- the amount of money to be transferred;

- the verb that identifies the financial transaction (either "send" or "demand"); and

- the identification of PosdataP2P service at the social channel.

Fig. 1 shows an example of the three text messages required to carry out a payment using the Facebook channel. First, Bob sends a post to make a payment of $4.5 €$ to his friend Alice. Then, the PosdataP2P service requires Bob to confirm the transaction and authorize it by using a second authentication factor and channel. Finally, the service notifies Bob (and Alice, not shown in Fig. 1) when the transaction is completed.

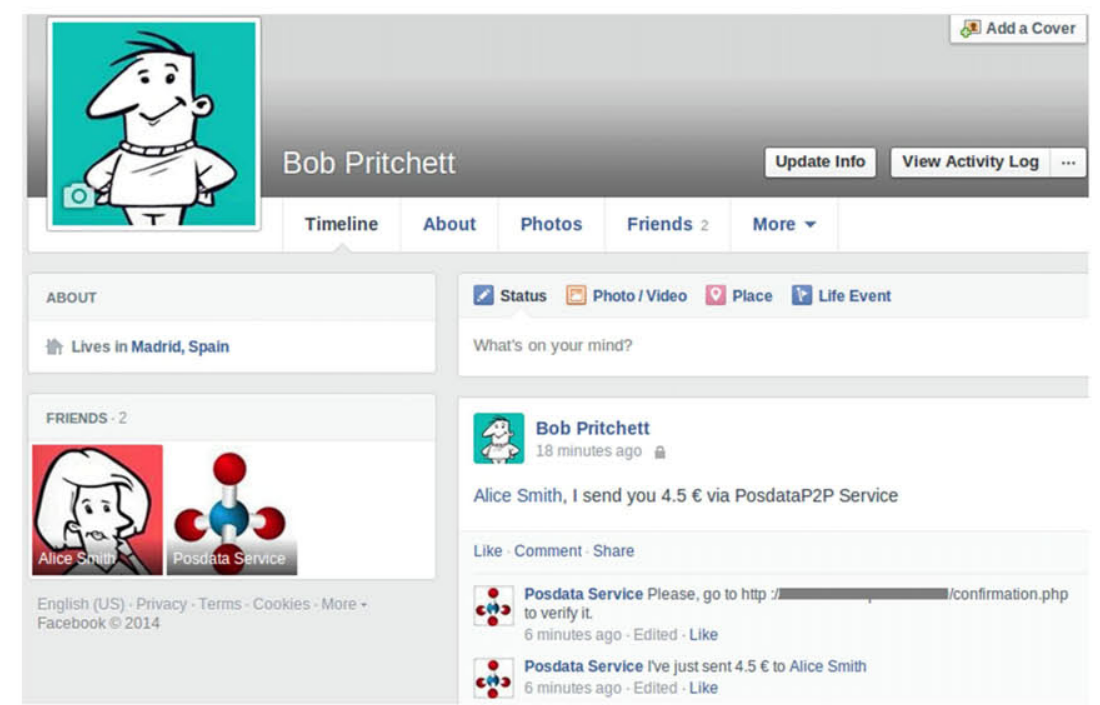

Fig. 1. Screenshot of the PosdataP2P service using Facebook as a channel.

Interestingly, the use of the PosdataP2P service generates financial data regarding USC holders, which properly combined with social information from the holder's 
online social network can be used to build a model intended to improve and personalize USC-based services. However, gathering information from disparate sources and integrating it to produce knowledge about users entails a set of challenges, which are discussed in the following sections.

\section{From Personal Data to User Models}

Personal data can be defined as the digital data created by and about one person [1]. There are many taxonomies of personal data, but we will consider the proposed by the Organization for Economic Co-operation and Development (OCDE) in [6], which categorizes data according to the manner in which they were originated:

- Provided: The data are originated from direct actions taken by the data subject, whereby they are fully aware of the actions that lead to data origination. For example, as a USC holder fills in the PosdataP2P service registration form, he is willingly giving demographic data and expressing some of his preferences.

- Observed: The data are captured by others and recorded in a digital format. For example, data regarding the payments among USC holders are captured by the PosdataP2P service.

- Derived: The data are generated from other data, and are not based on probabilistic reasoning. Examples of these data are classifications based on existing data such as active customer for those users that transfer or request amounts of money over a given, computed threshold, and during a defined time period.

- Inferred: They are created by a probability-based processing of any of the previous data, such as a credit risk score.

All this set of personal data are denoted as "identity", "user profile", or "user model" depending on the application area. Although these terms are closely interrelated (Fig. 2), and are used as synonyms many times, there are subtle differences between them.

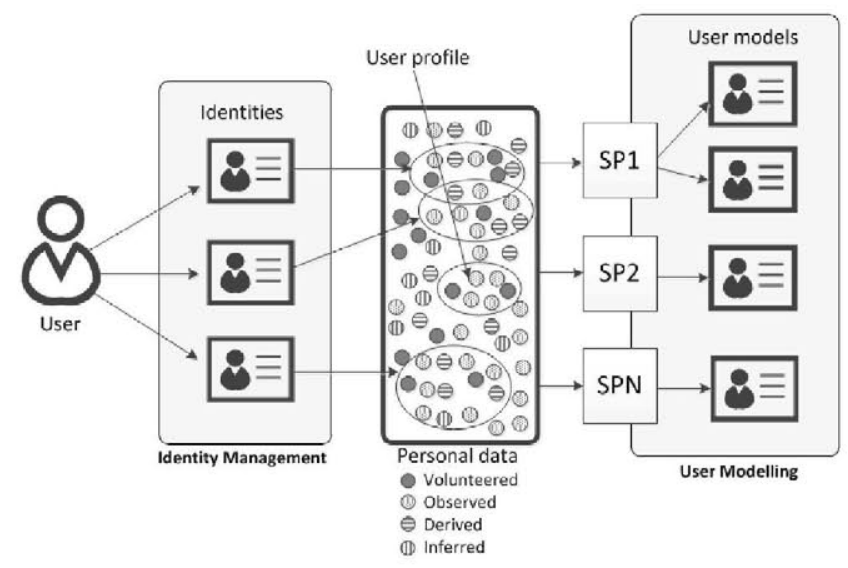

Fig. 2. Relationships between user identity, profile, and model. 
A digital identity broadly refers to the set of permanent or long-lived temporal attributes associated with an individual [7]. However, although it might contain all provided, observed, derived, and inferred data, its focus is on the subset of data that allows identifying the individual within a domain. Correspondingly, identity management functions and capacities guarantee the person identity while supporting business and security applications, e.g. authentication, authorization, single sign-on, etc.

On the other hand, the terms user profile and user model originate in the HumanComputer Interaction (HCI) area. Taking the differentiation that many authors have done [8] as the starting point, we can define a user profile as a collection of provided and/or observed data about a person. That is, a user profile contains raw personal data, without any processing or interpretation. Depending on the business goals and the amount of personal data that a user profile contains, it can be processed, modelled and interpreted to obtain a user model. This process is called user modelling.

As a result, a user model is understood as the interpretation of a person in a specific context for an organization. It includes what the organization thinks the user is, prefers, wants, or is going to do, and comprises mainly derived and inferred data but can also include volunteered or observed data. The user model can be used to predict user behaviour, recommend new contents or services, or personalize user interaction, among others. In the end, it represents a core business element: improving user experience and engaging users; therefore, organizations can provide better services. These improvements may turn into competitive advantages and economic benefits.

User modelling process covers different stages that can take place cyclically [9]. First, a data collection stage analyses what, how, and where the personal data is obtained. Then, a processing stage creates relevant knowledge with the previous data and produces a user model. Finally, the user model is applied to some business process, sometimes generating new personal data as a result, which may feedback the first stage closing the loop.

\section{Challenges in the User Modelling Process}

Traditionally, user modelling is a one-sided process where service providers autonomously collect personal data and then generate user models that satisfy their business objectives in a specific domain. As time passed, it emerged the need of sharing, interchanging, and eventually reusing personal data and user models (or parts of them) between service providers. This process entails a set of challenges such as the means to retrieve and share information from different sources, how to build a user model from raw data, and how to represent it. Moreover, given that it involves the use of sensitive information, users have to be included in the user modelling process. They need tools and mechanisms to manage and control their flows of personal data, and to support the setting of privacy preferences to be automatically enforced at the request of information. In the following subsections, we describe and discuss the most representative challenges for our work. 


\subsection{Data provision approach}

The production of user profiles and models requires a first process of provision and collection of personal data. Nowadays, personal data are distributed across different service providers that are responsible for storing and managing them. This traditionally entailed that each service provider possessed and used its own personal data, but this has changed over time.

In the online social context, all the mainstream social networks provide Application Program Interfaces (APIs) and web infrastructure for third parties to gather and modify users' personal information. For example, the Facebook Graph API [10] allows third parties to gain access to Facebook users' information through a HTTPbased API, the same as Twitter does with its own proprietary REST-based API [11].

To cope with the heterogeneity of personal and social information sharing on the Web some initiatives have come about such as OpenSocial [12]. It includes a set of open APIs that developers can use to gain access to users' personal resources hosted by different providers that have implemented them, for example, Google or LinkedIn.

\subsection{Personal Data Representation}

One of the most problematic issues related to the use, sharing and interchanging of personal data is to identify what elements are needed and how they are represented from a syntactic and semantic perspective.

The variety of personal data types is huge, probably as big as service providers, business needs and application domains. On the other hand, there are too many proposals of standards and proprietary solutions to represent each personal data category. For example, as regards social information about users, different standards have been proposed such as XHTML Friends Network (XFN), Family Tree Markup Language (FTML) or Friend of a Friend (FOAF) [13], in addition to the proprietary solutions used by different social networks providers.

As a result, the diversity of personal data representations raises the challenge of choosing the representation technique that better fits the organization needs. There are a lot of techniques that have been used over the time such as a feature-based pairs (or vector-space solutions), history-based models, user-rating matrix, etc. One of the most beneficial possibilities has been the use of ontologies, as they provide formal semantics, facilitation of reuse and portability, and allows the automation and use of inference engines to obtain user models [14]. It is important to note that when there is a description of a domain, represented by an ontology, an individual user model can be represented as a partial graph of the concepts [15].

\subsection{Identity and Privacy Management}

Other issues related to sharing personal data between different administrative domains is the proper authentication of the users in each domain, the management of the authorizations to gain access to protected resources, and the protection of the users' privacy rights. 
Identity management is the discipline that deals with these issues as it refers to the processes involved with the management and selective disclosure of user-related information either within an institution or between several of them, while preserving and enforcing privacy and security requirements [16]. Identity management on the Web has empowered users to gain control of the personal information that is exchanged between the parties involved, by considering user-centric architectural and usability aspects [17].

Nowadays, the delegation of the authorization model proposed by OAuth 2.0 [18] has become the cornerstone supporting identity management on the Web: All the Facebook, Twitter and other social-based APIs for personal information sharing rely on OAuth 2.0 to manage access to users' social information. The OAuth 2.0 framework introduces a third role to the traditional client-server authentication/authorization model: the resource owner. Following this model, the client (which is not the resource owner, but is acting on his/her behalf) requests access to resources controlled by the resource owner, but hosted by a container i.e. the online social network. OAuth 2.0 allows the social network provider to verify the identity of the client making the request, as well as ensuring that the resource owner has authorized the transaction without revealing their credentials.

Regarding privacy aspects, data protection laws of most countries as well as international privacy guidelines or directives [19], such as the OECD Privacy guidelines and the European Union Data Protection Directive, require basic privacy principles to be guaranteed when personal data (be it identity, user profile or user model) are collected or processed. Among others, these principles include: minimizing the routine collection and use of personally identifiable information; implementing user-centric approaches where users have the maximum control over their data; and providing privacy as the default option.

\section{The Personal Socio-Economic Network}

The PosdataP2P service provides users with new on-line banking channels (texting systems and popular social networks) that fit university students' habits. It processes texts from different social channels that are transformed into financial orders if applicable. This information can be collected and modelled to obtain a Personal SocioEconomic Network (PSEN) that represents the interchange of money between people.

In the following section, we present an overview of the PosdataP2P service architecture, detailing just those modules and processes required for the PSEN modelling process.

\subsection{General Architecture}

Fig. 3 represents the PosdataP2P architecture and its relations with the different social channels, Santander Bank infrastructure, and the user. We can distinguish seven main modules within PosdataP2P architecture: User Manager, Registrar, Listeners, Dispatchers, Analyser, and User Data Store. 


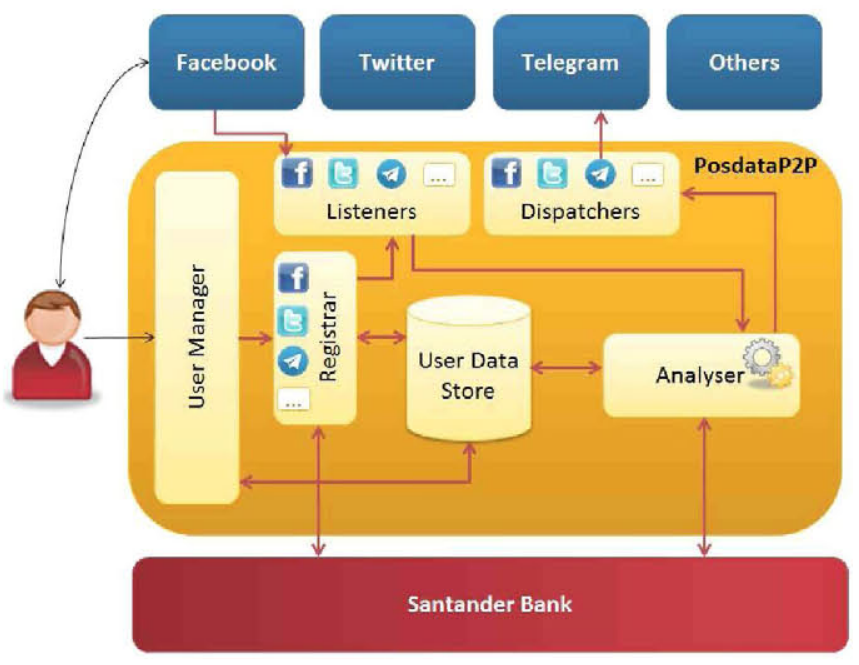

Fig. 3. PosdataP2P service architecture.

The User Manager allows users to interact with the PosdataP2P service to sign in, activate their social channels, and check and manage their data and user accounts. The User Data Store is the central entity where all the personal socio-economic information is stored. The Registrar is the module in charge of identity management interactions, enabling the communications with the user social channels and Santander Bank infrastructure.

The Listener comprises a set of components (one for each social channel) that are waiting for user texts that fit a predefined pattern where the PosdataP2P service is identified. Once a listener detects a user request it is redirected to the Analyser, which checks the validity of the user order, interacting with Santander Bank infrastructure to make payments if applicable. The Analyser is also in charge of communicating with the Dispatcher, which notifies users of the result of their orders or asks for extra information. Moreover, the Analyser processes and models the results of the transactions and stores them in the User Data Store.

User Model Feeding. The first process that we have considered in the production of the PSEN is the provision of personal data. This information can be obtained from three sources in the PosdataP2P service: the users themselves, their activation of social channels, and their use of the service.

Users provide volunteered information when they sign in to the PosdataP2P service through the User Manager. It contains several web pages and a main form that users fill in to provide personal data. Specifically, the service asks for user's first name, last name, gender, date of birth, email and USC number. Some of this information is redirected to the Registrar to certify, together with Santander Bank, the user identity.

Next, users can choose and activate their different social channels to make financial transactions. This step requires a dialog with the social channel provider, including an authentication process to confirm the user identity at the provider and an au- 
thorization mechanism to access, share and exchange personal data. At the end of the process, the PosdataP2P service only collects the user identifier of each social channel and the associated authorization tokens.

Finally, the use of the PosdataP2P service generates relevant information. In our case, the Analyser receives all the user requests for money transfers and demands.

All the information collected in the previous processes (sign in, social channels activation, and service use) are modelled and stored in the User Data Store module, making up the PSEN.

PSEN Representation. We have defined an ontology to model the PSEN, following the ontology development methodology proposed by [20]. It abstracts the main concepts from our PosdataP2P scenario. Furthermore, we have considered the reusing of existing ontologies, which is a must to allow semantic and syntactic interoperability. Thus, we have identified the FOAF ontology as the best alternative for representing people in a social network context and therefore, we have extended it to include the economic concepts.

FOAF is a Semantic Web vocabulary or specific-domain ontology of the area of social networks, managed by the FOAF project [21]. It includes the main terms to describe people, the links between them and the things they create and do. Among its concepts, it includes the Agent class to represent a person, organization, or a group. We have focused our definition of the PSEN on the Person class of FOAF, given the current functionalities of the PosdataP2P service.

A Person in FOAF includes a set of properties that allows us to model a user of the PosdataP2P service. Specifically, we have used the corresponding FOAF properties to describe the user's demographic information: firstName, lastName, gender, age, birthday, and mbox.

The mbox property represents an email address, and is usually used to identify a person. However, we identify a user in PosdataP2P by his USC number. On the other hand, given that each user has associated a set of social channels, we have modelled his identifiers in these channels. In this sense, we have made use of the OnlineAccount class of FOAF that allows the modelling of different web identities or online accounts of a person.

An online account includes two main properties in FOAF: accountName and accountServiceHomepage. The former is a textual representation of a unique identifier associated with the user account, while the accountServiceHomepage indicates the homepage of the service provider for this online account. We have related a person to different instances of the OnlineAccount class to represent the USC identification and each of the social channels activated by the user.

As regards the economic concepts that the PosdataP2P service manages, we have defined a main class called EconomicActivity. It represents the different economic transactions that can take place between people. To date, we have identified two main economic activities that have been defined as subclasses of the EconomicActivity class: Payment and Demand. The former indicates that a person has carried out a payment to another person; while the second one indicates that a person is asking for money to another person. 
Each economic activity has associated two principal properties: initiator and terminator. They associate an economic activity with two persons, representing who starts the activity and who is the objective of it, respectively. Thus, two persons are related by an EconomicActivity and a person can have several economic activities.

In addition to the previous one, the EconomicActivity class includes other three properties: money (relates the economic activity with a Money class to represent how much is being paid or demanded. The Money class includes the amount and type of the money, using Dbpedia information [22]); timestamp (indicates when the activity took place via a combination of date and time of day via a xsd:dataTime); and channel (indicates what social channel was used to carry out the economic activity such as Facebook, Twitter, etc.).

It is important to point out that FOAF, and specifically the Person class, includes a knows property to relate a person to another person. It takes the broadest view of "knows", meaning a reciprocal interaction between them but it does not imply a friendship or endorsement. In this sense, the PosdataP2P service creates knows relations when an economic activity between two persons is detected.

Fig. 4 symbolizes the definition of the EconomicActivity class and its relation to the Person class of FOAF. We have omitted the details of the Person properties to represent demographic user information but we include the new concepts defined in the PSEN.

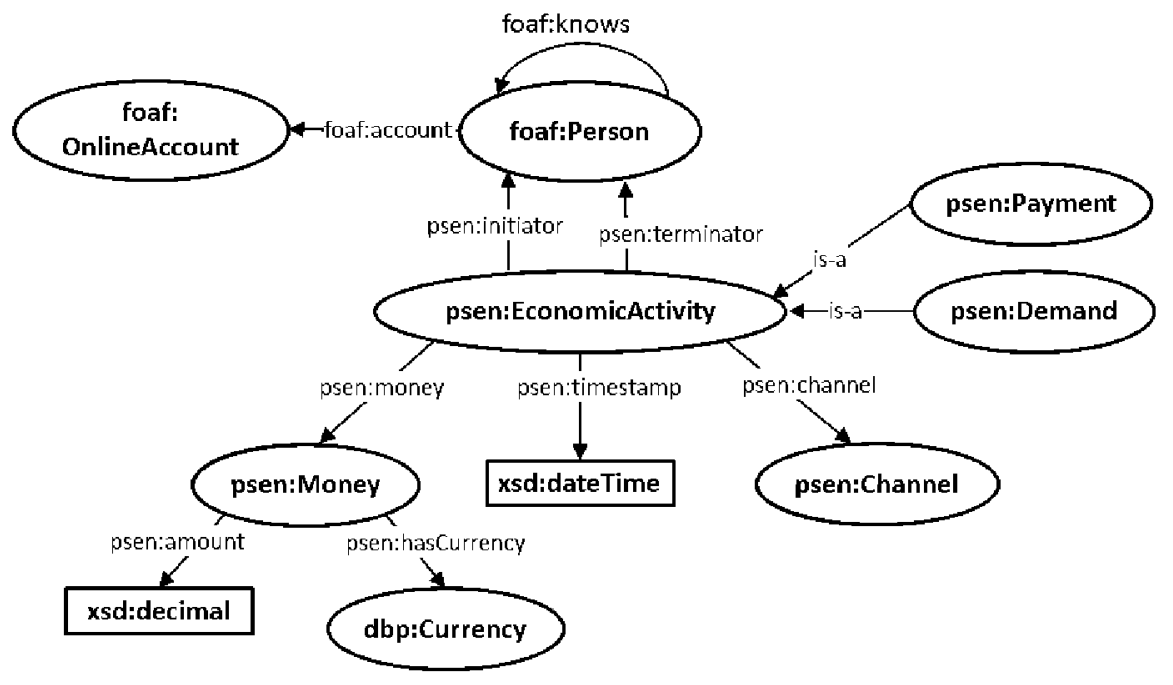

Fig. 4. Definition of an economic activity.

Identity and Privacy Management. The PosdataP2P service requires an identity management infrastructure to manage the different users' identities associated with the USC holder and each of his activated social channels. As we have seen before, OAuth 2.0 has become the standard to manage digital identities on the Web and thus, we have based our infrastructure in this standard, developing it in the Registrar. 
The process begins when the user chooses a social channel to be activated. The user is then redirected to the social channel provider site to grant our service the required level of authorization. If successful, the social channel provider delivers an authorization token that allows subsequent access to the user profile and information.

The PosdataP2P service has been designed to observe privacy-by-design principles and therefore, to guarantee explicit and conscious user governance of their personal information in a safe and easy way. In particular, the service reduces the personal information retrieved from external sources to the minimum necessary: Users' identification at the social channels, the credentials required by OAuth 2.0, and their posts to enable the PostdataP2P service. Furthermore, the PosdataP2P service allows its users to manage the sources of personal data comprehensibly from a central control point working as a digital identity dashboard. From there, users are able to manage their associated channels and carry out their access, object and cancel rights for the information stored.

\section{Implementation Details}

We have developed the infrastructure necessary to produce and manage the PSEN, within the PosdataP2P service prototype. First, web components have been developed to give shape to the PosdataP2P service and Santander Bank infrastructure including its main features: user registration, activation of social channels, and checking and management of user balance and PSEN information. Moreover, we have simulated Santander Bank functionalities to communicate with PosdataP2P service in the registration process and the carrying out of payments.

Additionally, we have implemented the Facebook channel to allow users to issue money transfers through simple text messages (specifically, posting predefined texts, see Fig. 1). Facebook provides developers with different products and APIs that allows building different functionalities and applications, and getting information from their users and pushing notifications to them. In this sense, we have developed a Facebook application that uses the Facebook Login and the Graph API.

The Facebook Login allows our application to link the Facebook user account with the PosdataP2P service. It implements a slight variant of OAuth 2.0 protocol for carrying out the authentication and authorization processes. We have used it to gather the Facebook user identifier and authorization token, and to activate a Facebook listener.

The Facebook listener is part of our Facebook application and has been implemented using the Graph API that allows developers to read and write to the Facebook user profiles. It includes a special feature called Real Time Updates that notifies about changes in a specific field. The data that an application can subscribe to is limited by Facebook policies, and with the notification that is sent, it is only possible to know that a field has been modified, but not the actual modification. This last part has been made by REST requests to Facebook's servers. The information that can be asked for and collected is mainly delimited by the user. It uses specific permissions in the login process to assure that the user knows what, when and who can read and manipulate their profile. 
As regards the PSEN modelling and storage, we have used Protégé [23] to define the ontology and Jena [24] to manage the population and storage of the PSEN instances. The former is a free, open-source ontology editor and a framework written in Java that supports Resource Description Framework (RDF) specifications and the latest Web Ontology Language (OWL 2) from the W3C. Protégé provides two main environments, a desktop tool and a web-based implementation, to create, upload, modify and share ontologies. We have used the desktop solution that is quite intuitive and allows us to import FOAF and create our PSEN ontology easily.

On the other hand, the PSEN development environment has been implemented with Jena. It is also a free, open-source Java framework that provides all the necessary components for creating and reading Resource Description Framework (RDF) graphs, working with models based on RDF Schema and Web Ontology Language (OWL), querying RDF through SPARQL, and reasoning over the stored data to expand and check the content.

\section{$7 \quad$ Related Work}

As we have seen above, user model creation involves four main stages: data collection, modelling and application, in addition to user involvement to manage and control their flows of personal data. These ones have been widely studied and analysed over the time and we can find related literature, either addressing one of them in depth or proposing particular solutions for some of them in different application domains. For example, [25] describes the evolution of specialised systems, namely Generic User Modelling Systems, that tackle the different user modelling stages; [15] presents the user modelling techniques, its challenges and the state-of-the-art research, focusing on ubiquitous environments; and [26] gives an overview of user modelling in connection with the semantic technologies.

Considering the social network context, there are many efforts that cover the user modelling stages, either focusing on a single social network or integrating information from some of them. For example, [27] obtains Facebook data for recommendation processes; [28] retrieves user interactions from Facebook to produce behavioural patterns; [29] proposes a model to capture social relationships and status from different social media contexts; or [30] describes how to aggregate different user profiles from several social networks. The study presented in [31] includes an in depth comparison of the user modelling strategies in a multi-social context.

Our work is aligned with these last solutions, fitting and contributing to user modelling in a multi-social network context. Unlike the other proposals, we have focused our efforts on creating a relation to the personal financial sector. To the best of our knowledge, this is the first effort to integrate social and economic data aiming at modelling users. 


\section{Conclusions and Future Work}

In this paper we have presented our approaches and contributions to modelling a personal socio-economic network that includes the economic activities carried out between people in social contexts. Our work has been focused on the collection of personal data from several sources, and the modelling (including the representation) of this information. For that, we have leveraged on an innovative service, namely PosdataP2P, developed in the context of a Santander Bank research initiative.

The PosdataP2P service allows users to make or demand payments to their friends through simple text messages, using different social channels such as Facebook, Twitter and Telegram. It produces a set of relevant information that we have collected and stored ontologically to model the personal socio-economic network. This advanced approach tackles a set of ambitious tasks of integrating data from diverse sources, identity management, and representation of new knowledge, which we have described.

Our future work includes exploring and designing the elements required by the PosdataP2P service to allow third parties to retrieve custom-made user models from the personal socio-economic network, to better fit the business needs of the requesting parties. These models will comprise personalized social-economic networks or inferred values for a person such as all of his payments or debts, the total amount of money exchanged, or the friends' list that he interacts more with.

Acknowledgments. This work is part of the Center for Open Middleware (COM), a joint technology center created by Universidad Politécnica de Madrid, Banco Santander and its technological divisions ISBAN and PRODUBAN.

\section{References}

1. Schwab, K., Marcus, A., Oyola, J. R., Hoffman, W.: Personal Data: The Emergence of a New Asset Class. World Economic Forum (2011)

2. Gonzalez, F.: Banks need to take on Amazon and Google or die. Financial Times (2013)

3. Auerbach, P., Argimon, R.F., Hieronimus, F., Roland, C., Teschke, B.: Banking on Customer Centricity - Transforming Banks into Customer-Centric Organizations. EMEA Banking Practice, McKinsey \& Company (2012)

4. Chaia, A., Dalal, A., Goland, T., Gonzalez, M. J., Morduch, J., Schiff, R.: Half the World is Unbanked. Financial Access Initiative Framming Note (2009)

5. Observatorio TUI, International University Smart Card Research Centre, http://en.observatoriotui.com/home

6. Working Party on Security and Privacy in the Digital Economy: Protecting Privacy in a Data-driven Economy: Taking Stock of Current Thinking. Organisation for Economic Cooperation and Development (2014)

7. Windley, P.: Digital Identity. O'Reilly Media Inc. (2005)

8. Fröschl, C.: User Modeling and User Profiling in Adaptive E-learning Systems. Master's Thesis, Graz University of Technology (2005) 
9. Barla, M.: Towards Social-based User Modeling and Personalization. Dissertation Thesis, Slovak University of Technology in Bratislava (2014)

10. Facebook Developers, The Graph API, https://developers.facebook.com/docs/graph-api

11. Twitter Developers, https://dev.twitter.com/

12. OpenSocial, http://opensocial.org/

13. Nabeth, T.: D2.3: Models. Deliverable, Future of Identity in the Information Society Consortium (2005)

14. Sosnovsky, S.: Ontological Technologies for User Modeling. Committee of the School of Information Sciences, University of Pittsburgh (2008)

15. Kuflik, T. Kay, J., Kummerfeld, B.: Challenges and Solutions of Ubiquitous User Modeling. In: Krüger, A., Kuflik, T.: Ubiquitous Display Environments, pp. 7-30. Springer, Berlin Heidelberg (2012)

16. Álamo, J. M., Fernández, A. M., Trapero, R., Yelmo, J. C., Monjas, M. A.: A PrivacyConsiderate Framework for Identity Management in Mobile Services. Journal Mobile Networks and Applications 16 (4), 446-459 (2011)

17. Bhargav-Spantzely, A., Camenisch, J., Gross, T., Sommer, D.: User centricity: A taxonomy and open issues. Journal of Computer Security - The Second ACM Workshop on Digital Identity Management 15 (5), 493-527 (2007)

18. The OAuth 2.0 Authorization Framework, https://tools.ietf.org/html/rfc6749

19. Wright, D., Raabb, C.: Privacy principles, risks and harms. International Review of Law, Computers \& Technology, 28 (3) (2014)

20. Noy, N. F., McGuinness, D. L.: Ontology Development 101: A Guide to Creating Your First Ontology. Technical report, Stanford Knowledge Systems Laboratory (2001)

21. Foaf project, http://www.foaf-project.org/

22. DBpedia, http://dbpedia.org/About

23. Protégé, http://protege.stanford.edu

24. Apache Jena, https://jena.apache.org/

25. Kobsa, A.: Generic User Modeling Systems. The Adaptive Web Lecture Notes in Computer Science 4321, 136-154 (2007)

26. Aroyo, L., Houben, G. J.: User Modeling and The Adaptive Semantic Web. Journal Semantic Web 1, 1-6 (2010)

27. Shapira, B., Rokach, L., Freilikhman, S.: Facebook single and cross domain data for recommendation systems. Journal User Modeling and User-Adapted Interaction, 23(2-3), 211-247 (2013)

28. Ortigosa, A., Quiroga, J.I., Carro, R.M.: Inferring user personality in social networks: A case study in Facebook. In: 11th International Conference on Intelligent Systems Design and Applications, pp. 563-568, IEEE, New York (2011)

29. Kabir, M. A., Han, J., Yu, J.: Alan Colman User-centric social context information management: an ontology-based approach and platform. Personal and Ubiquitous Computing, $18(5), 1061-1083(2014)$

30. Orlandi, F., Breslin, J., Passant, A.: Aggregated, interoperable and multi-domain user profiles for the social web. In: 8th International Conference on Semantic Systems, pp. 41-48, ACM, New York (2012)

31. Abel, F., Herder, E., Houben, G.J., Henze, N., Krause, D.: Cross-system user modeling and personalization on the Social Web. User Modeling and User-Adapted Interaction, 23(2-3), 169-209 (2013) 